\title{
Repressing PTBP1 is incapable to convert reactive astrocytes to dopaminergic neurons in a mouse model of Parkinson's disease
}

\section{Authors:}

Weizhao Chen ${ }^{1,2} \dagger$, Qiongping Zheng ${ }^{1,2} \dagger$, Qiaoying Huang ${ }^{1,2}$, Shanshan $\mathrm{Ma}^{1,2^{*}}$, Mingtao $\mathrm{Li}^{1,2^{*}}$

\author{
Affiliation: \\ Sun Yat-sen University, Guangzhou, Guangdong, China, 510080 \\ Guangdong, China, 510080 \\ $\dagger$ Indicates equal contribution. ${ }^{*}$ To whom correspondence should be addressed at \\ mashsh3@mail.sysu.edu.cn (S.Ma),limt@mail.sysu.edu.cn (M. Li)
}

${ }^{1}$ Guangdong Provincial Key Laboratory of Brain Function and Disease, Zhongshan School of Medicine,

${ }^{2}$ Department of Pharmacology, Zhongshan School of Medicine, Sun Yat-sen University, Guangzhou,

\section{Abstract}

Lineage reprograming of resident glia cells to induced dopaminergic neurons (iDAns) holds attractive prospect for cell-replacement therapy of Parkinson's disease (PD). Recently, whether repressing polypyrimidine tract binding protein 1 (PTBP1) could truly achieve efficient astrocyte-to-iDAn conversion in substantia nigra and striatum aroused widespread controversy. Although reporter positive iDAns were observed by two groups after delivering adeno-associated virus (AAV) expressing a reporter with shRNA or Crispr-CasRx to repress astroglial PTBP1, the possibility of AAV leaking into endogenous DAns could not be excluded without using a reliable lineage tracing method. By adopting stringent lineage tracing strategy, two other studies showed that neither knockdown nor genetic deletion of quiescent astroglial PTBP1 fails to obtain iDAns under physiological condition. However, the role of reactive astrocyte might be underestimated since upon brain injury, reactive astrocyte could acquire certain stem cell hallmarks which may facilitate the lineage conversion process. Therefore, whether reactive astrocytes could be 
genuinely converted to DAns after PTBP1 repression in a PD model needs further validation. In this study, we used Aldh1l1-CreER ${ }^{T 2}$-mediated specific astrocyte-lineage tracing method to investigate whether reactive astrocytes could be converted to DAns in the 6-OHDA PD model. However, we found that no astrocyte-originated DAn was generated after effective knockdown of astroglial PTBP1 either in the substantia nigra or in the striatum, while AAV "leakage" to nearby neurons was observed. Our results further confirmed that repressing PTBP1 is unable to convert astrocytes to DAns no matter in physiological or PD-related pathological conditions.

Key words: lineage reprogramming, astrocyte-to-iDAn conversion, PD, PTBP1, AAV leakage, knockdown, conditional deletion, lineage tracing, quiescent astrocyte, reactive astrocyte, 6-OHDA model

\section{INTRODUCTION}

The emergence and fast development of in vivo cell reprogramming technology converting deleterious astrocytes to functional neurons holds great promise for neuroregenerative therapy(Torper and Gotz, 2017). Various groups around the world had successfully achieved astrocyte-to-neuron (AtoN) conversion by forced expression of different pro-neural transcription factors (TF) such as Ngn2(Grande et al., 2013), Ascl1(Liu et al., 2015), NeuroD1(Guo et al., 2014), SOX2(Niu et al., 2013) or various TF combinations(Rivetti di Val Cervo et al., 2017; Wu et al., 2020; Lentini et al., 2021). Differing from the TF over-expression approach, through repressing an RNA-binding protein-PTBP1, two groups respectively reported that functional neurons including DAns could be induced from astrocyte rapidly and efficiently in vivo, reconstructing the nigrostriatal circuit and improving motor deficit in a mouse PD model(Qian et al., 2020; Zhou et al., 2020).

Nevertheless, without substantiating the exact origin of the nascent iDAns using reliable lineage 
tracing strategy, these two outstanding works soon aroused widespread debate and argument (Arenas, 2020;

Jiang et al., 2021; Qian et al., 2021). Most recently, by adopting stringent lineage tracing method, two studies arguing against previous findings were published. One group showed that AAV-shPtbpl-induced, presumed astrocyte-converted iDAns were not truly transdifferentiated from astrocytes but merely AAV-infected endogenous neurons due to virus leakage(Wang et al., 2021). Another group reported that no astrocyte-derived neuron including DAn was generated in multiple brain regions including substantia nigra and striatum in astrocyte-specific Ptbpl deletion mice. (Blackshaw et al., 2021). However, both studies simply focused on quiescent astrocytes instead of reactive astrocytes and whether reactive astrocytes could be converted to neurons more effectively after PTBP1 repression needs further verification.

During brain injury or neurodegenerative disease, astrocytes become activated and acquire certain characteristics of neural stem cells (NSCs) such as proliferative, Nestin- or Vimentin-immunoreactive and even multipotent(Buffo et al., 2008; Robel et al., 2011; Shimada et al., 2012; Sirko et al., 2013). Some researchers further claimed that reactive astrocytes with stem cell hallmarks could be reprogrammed to neurons more easily and more efficiently than quiescent astrocytes(Grande et al., 2013; Guo et al., 2014; Wan et al., 2014; Brulet et al., 2017; Mattugini et al., 2019). Therefore, we adopted 6-hydroxydopamine (6-OHDA) PD model with lineage tracing method to investigate whether reactive astrocytes could truly be converted to neurons including DAns.

\section{Materials and Methods}

\section{Animals}

All animal experiments were performed in accordance with the guidelines of the Institutional Animal Care and Use Committee of University. The protocol was reviewed and approved by the Ethics Committee 
on Laboratory Animal Care. The mice were housed in rooms with controlled 12 hours light/dark cycles, temperature, and humidity, and food and water were provided ad libitum. Eight- to ten-week-old C57BL/6 mice weighing 22-26g were obtained from the Beijing Vital River Laboratory Animal Technological Company (Beijing, China). Aldh1l1-CreER $R^{T 2}$ transgenic mice and $R p l 22^{H A / H A}$ mice (Ribotag) were obtained from The Jackson Laboratory (Stock number \#029655, \#011029). Hemizygous Aldh1l1-CreER ${ }^{T 2}$ males or females were used for breeding to the $R p l 22^{H A / H A}$ mice. Aldh $111-C r e E R^{T 2}$ : Ribotag mice aged at 8-10 weeks were used for lineage tracing experiments.

\section{Tamoxifen(TAM) administration}

The protocol of TAM administration was determined according to previous work(Srinivasan et al., 2016) with little modifications. Briefly, TAM free-base (Sigma, China) was dissolved in corn oil (Aladdin, China) at a concentration of $10 \mathrm{mg} / \mathrm{mL}$ in a $60^{\circ} \mathrm{C}$ water-bath for 30 minutes. TAM was orally administered at a daily dose of $100 \mathrm{mg} / \mathrm{kg}$ body weight for five consecutive days. Experiments were performed two weeks after the last TAM administration.

\section{6-OHDA model}

The procedure was based on previous study with few modifications(Rivetti di Val Cervo et al., 2017; Qian et al., 2020; Zhou et al., 2020). In brief, 6-OHDA (Sigma, USA) was dissolved in ice-cold saline solution $(0.9 \% \mathrm{NaCl})$ containing $0.2 \mathrm{mg} / \mathrm{mL}$ L-Ascorbic acid (BBI Life Sciences, China) at a concentration of $3 \mathrm{mg} / \mathrm{mL}$. Mice were anesthetized with 3\% isoflurane and then placed in a stereotaxic instrument (Model 940, Kopf Instruments, USA). After anesthesia, mice were delivered with $1 \mu \mathrm{L}$ of 6-OHDA solution ( $3 \mathrm{mg}$ ) into the right medial forebrain bundle $(\mathrm{mFB})$ at $100 \mathrm{~nL} / \mathrm{min}$ according to the following coordinates: 
anteroposterior $(\mathrm{A} / \mathrm{P})=-1.20 \mathrm{~mm}$, mediolateral $(\mathrm{M} / \mathrm{L})=1.30 \mathrm{~mm}$, dorsoventral $(\mathrm{D} / \mathrm{V})=-4.75 \mathrm{~mm}$. Injections were conducted with a $10 \mu \mathrm{L}$ syringe connected to a 33-Ga needle (Hamilton, USA) using a microsyringe pump (KDS LegatoTM 130, USA). After 6-OHDA injection, mice were typically allowed to recover for three weeks with intense daily care.

\section{AAV production and infection}

AAV2/5-hGFAP-EGFP-5'miR-30a-shRNA(Ptbp1)-3'miR-30a-WPREs (AAV-shPtbpl, $3.41 \times 10^{12}$ $\mathrm{vg} / \mathrm{mL}$ ) and the control virus AAV2/5-hGFAP-EGFP-5'miR-30a-shRNA(scramble)-3'miR-30a-WPREs (AAV-shscramble, $2.57 \times 10^{12} \mathrm{vg} / \mathrm{mL}$ ) were synthesized based on the pAAV- $h G F A P$-EGFP-WPRE-hGH plasmid (Addgene \# 105549) and packaged by Brain VTA (Wuhan, China). For PTBP1 repression, we used the same mouse Ptbpl target sequence(5'-GGGTGAAGATCCTGTTCAATA-3') as previously reported (Qian et al., 2020) while AAV-shscramble expressing scramble shRNA (same nucleotide composition but not in a same sequence order) was used as control.

Before injection into the mouse brain, the AAVs were adjusted to $1 \times 10^{12} \mathrm{vg} / \mathrm{mL}$ using sterile Dulbecco's Phosphate Buffered Saline (DPBS, Gibco, USA). Three weeks after 6-OHDA lesion, mice were subjected to AAV injection into the substantia nigra $(1 \mu \mathrm{L})$ or the striatum $(2 \mu \mathrm{L})$, respectively, at a speed of $100 \mathrm{~nL} / \mathrm{min}$. The coordinates indicating distance $(\mathrm{mm})$ from bregma were $\mathrm{A} / \mathrm{P}=-2.90 \mathrm{~mm}, \mathrm{M} / \mathrm{L}=1.30 \mathrm{~mm}$, and $\mathrm{D} / \mathrm{V}=-4.35 \mathrm{~mm}$ for the substantia nigra, and $\mathrm{A} / \mathrm{P}=0.80 \mathrm{~mm}, \mathrm{M} / \mathrm{L}=1.60 \mathrm{~mm}$, and $\mathrm{D} / \mathrm{V}=-2.80 \mathrm{~mm}$ for the striatum. After injection, the needle remained in place for at least five minutes to prevent retrograde flow along the needle track and the needle was slowly removed from the mouse brain. Cleaning and suturing of the wound were performed after the needle was removed. 


\section{Immunofluorescent analysis}

For immunofluorescent analysis, mice were anesthetized with $1.25 \%$ Avertin and were perfused intracardially with ice-cold phosphate buffered saline (PBS), followed by $4 \%$ paraformaldehyde (PFA, Sigma, China) in PBS at a flow rate of $10 \mathrm{~mL} / \mathrm{min}$. The brains were then removed and post-fixed in $4 \%$ PFA at $4{ }^{\circ} \mathrm{C}$ overnight ( $8-12$ hours), followed by immersion in $20 \%$ and $30 \%$ sucrose for 24 hours respectively. Immunofluorescent analysis was performed as previously described (Yu et al., 2018; Hu et al., 2019). In brief, cryostat-coronal sections encompassing the entire midbrain $(20 \mu \mathrm{m})$ and the striatum(30 $\mu \mathrm{m}$ ) were serially collected. Free-floating sections were pre-incubated in blocking solution containing $5 \%$ normal donkey serum and $0.3 \%$ Triton $\mathrm{X}-100$ in $50 \mathrm{mM}$ Tris-buffered saline (TBS, $\mathrm{pH}=7.4$ ) at room temperature for $1 \mathrm{~h}$. Primary antibodies against HA tag (Rabbit, Abcam, ab9110, 1:1000), TH (Chicken, Millipore, AB152, 1:1000), NeuN (Mouse IgG1, Millipore, MAB377, 1:1000) and PTBP1 (Rabbit, Invitrogen, PA581297, 1:1000) were dissolved in diluent and incubated with sections overnight at $4^{\circ} \mathrm{C}$. After washing three times, sections were incubated with the secondary antibodies (Thermo Fisher or Jackson ImmunoResearch), which were conjugated with Alexa 488, Alexa 555 or Alexa 647 at room temperature for $1 \mathrm{~h}$. Finally, the sections were visualized under a confocal laser scanning microscope (LSM 780, Carl Zeiss, Germany).

\section{Statistics}

GraphPad Prism version 8.0 was used for statistical analysis. All data were presented as mean \pm SEM.

Paired-samples $\mathrm{t}$ test was used to determine the statistical significance $(p<0.05)$.

\section{Results}




\section{Repressing PTBP1 rapidly and efficiently induces viral-reporter-labeled neurons.}

To effectively repress astroglial PTBP1 in vivo, we designed and synthesized AAV (serotype 2/5) expressing EGFP followed shRNA targeting mouse Ptbpl as reported(Qian et al., 2020), under $h G F A P$ promoter (AAV-shPtbp1), which aimed for specific astrocytes targeting. A corresponding virus expressing scramble shRNA (AAV-shscramble) was used as control (Fig.1A).

In order to investigate whether repressing astroglial PTBP1 could gradually convert astrocytes to DAns in the substantia nigra and the striatum, brain slices of different time points (1,2 and 3 months) after AAV injection were collected for analysis. PTBP1 expression was not affected by AAV-shscramble (Fig. 1B upper panel) while it was downregulated to undetectable level by AAV-shPtbpl in $\mathrm{GFAP}^{+} \mathrm{GFP}^{+}$cells (Fig. 1B lower panel) from 1 month to 3 months, indicating astroglial PTBP1 was consistently repressed.

Pan-neuronal marker Neuronal Nuclei (NeuN) and DAn marker Tyrosine Hydroxylase (TH) were then co-stained with GFP respectively. The results showed that very few $\mathrm{GFP}^{+} \mathrm{NeuN}^{+}$cells (approximately 1-2\%) were detected even at 3 months after AAV-shscramble injection, while remarkable $\mathrm{GFP}^{+} \mathrm{NeuN}^{+}$cells were detected, with $12 \%, 27 \%$ and $46 \% \mathrm{GFP}^{+}$cells expressed $\mathrm{NeuN}$, among them $9 \%, 20 \%$ and $28 \%$ expressed TH (Fig. 1C), at 1, 2 and 3 months after AAV-shPtbpl injection in the substantial nigra. The gradual increase in numbers of $\mathrm{GFP}^{+} \mathrm{NeuN}^{+}$cells in AAV-shPtbpl injected in striatum was similar to that in the substantial nigra. But no $\mathrm{GFP}^{+} \mathrm{TH}^{+}$cell was found in the striatum. These results highly resemble Fu's study(Qian et al., 2020) but against Yang's study(Zhou et al., 2020).

However, all these results are not sufficient to prove that astrocytes were truly converted to neurons or DAns as recently reported AAV-mediated gene expression leakage into neuron(Wang et al., 2021).Thus, more solid evidence is needed to verify the exact origin of the viral-reporter-labeled neurons. 


\section{PTBP1 repression fails to convert quiescent astrocytes to DAns}

Genetic lineage tracing(Kretzschmar and Watt, 2012) has been widely recognized as the most convincing strategy for cell source identification, generally performed by combining a cell-specific Cre recombinase expressing mice with a Cre-activated reporter mice. Aldh1l1-CreER ${ }^{T 2}$ mice with highest specificity to target astrocytes(Srinivasan et al., 2016) were chosen to cross bred with a reporter mice $R p l 22^{H A / H A}$ (Ribotag)(Sanz et al., 2009), whose endogenous ribosomal protein Rpl22 is tagged with three copies of the hemagglutinin (HA) epitope after Cre-mediated recombination (Fig. 2A). After TAM-mediated induction of $\mathrm{CreER}^{\mathrm{T} 2}$ activity, over 99\% Aldolase C (AldoC) positive astrocytes were specifically labeled with HA epitope (Fig. 2B) and almost no HA leaky expression in neuron in the substantial nigra and the striatum of Aldh1ll-CreER ${ }^{T 2}$ :Ribotag mice.

Two weeks after TAM induction, Aldh1l1-CreER ${ }^{T 2}$ :Ribotag mice were injected with AAV-shPtbp1 into the substantia nigra or the striatum to verify whether the $\mathrm{GFP}^{+} \mathrm{TH}^{+}$or $\mathrm{GFP}^{+} \mathrm{NeuN}^{+}$cells were originated from HA-labeled astrocytes. Three months later, mice were sacrificed for triple immunostaining of GFP, HA and NeuN or GFP, HA and TH. Through exhaustive examination of the whole midbrain and striatum, we couldn't find any $\mathrm{GFP}^{+} \mathrm{TH}^{+}$or $\mathrm{GFP}^{+} \mathrm{NeuN}^{+}$cells that were simultaneously HA-positive(Fig. 2C), suggesting they were not converted from astrocytes but probably endogenous neurons infected with $\mathrm{AAV}$ and expressing GFP reporter.

Therefore, the above results clearly illustrated that PTBP1 repression fails to convert quiescent astrocytes to neurons including DAns, which is consistent with recent studies(Blackshaw et al., 2021; Wang et al., 2021). 
A lot of evidence has suggested that reactive astrocytes may acquire certain characteristics of NSC upon brain injury, which could promote AtoN conversion(Grande et al., 2013; Guo et al., 2014; Wan et al., 2014; Brulet et al., 2017; Mattugini et al., 2019). To verify whether repression PTBP1 could converts reactive astrocyte to neuron including DAns, we performed 6-OHDA lesion in the $\mathrm{mFB}$ on Aldh1l1-CreER ${ }^{T 2}$ :Ribotag mice 2 weeks after TAM administration.

Three weeks after 6-OHDA lesion in $\mathrm{mFB}$, the mice were subjected to AAV-shscramble or AAV-shPtbpl injection in the substantia nigra and the striatum respectively, and then sacrificed for immunostaining after three months (Fig. 3A).

Our results showed that 6-OHDA induced severe lesion to the nigrostriatal pathway with significantly reduced the number of DAns in substantia nigra and the densities of $\mathrm{TH}^{+}$fibers in the striatum. Meanwhile, astrocytes become remarkably activated, indicated by classic cytoskeletal and morphological changes including hypertrophy of the main processes and cell body and upregulation of intermediate filament proteins such as GFAP (Fig. 3B). However, no $\mathrm{HA}^{+}$cells co-expressing NeuN or TH could be detected either in the substantia nigra or the striatum (Fig. 3C \& D), no morphologic change of astrocytes could be seen after PTBP1 repression (Fig. 3C \& E) and only subtle changes in gene expression could be seen after astroglial Ptbp1 deletion(Blackshaw et al., 2021), suggesting that neither AtoN nor astrocyte-to-DAn conversion occurred. Together, these data demonstrate that repressing PTBP1 is also incapable to generate DAns from reactive astrocytes in a murine PD model.

In summary, we provide solid and convincing evidence that repressing astroglial PTB using shRNA-based RNA interference (RNAi) technology is unable to generate DAns from astrocytes neither in quiescent nor in reactive state. 


\section{Discussion}

In summary, we provide solid and convincing evidence that repressing astroglial PTBP1 using shRNA-based RNA interference (RNAi) technology is unable to generate DAns from astrocytes neither in quiescent nor in reactive state.

\section{Factors that lead to artificial AtoN conversion}

It is well known that excessive dosage of AAV increases the tendency of virus leakage to other cells nearby. Once the astrocyte-specific labeling reporter leaks to neuron, the reporter ${ }^{+}$neuron would then be misidentified as neuron converted from astrocyte, resulting false-positive results. In addition to virus dosage, other factors that may promote the artificial AtoN conversion in a previous study(Qian et al., 2020) will be discussed below.

Firstly, it is widely accepted that Gfap promoter, either employed in transgenic mice or in virus, is not stringent for astrocyte specific labeling and genetic manipulation. Various studies reported that a large proportion of neuron also has Gfap promoter activity especially after brain injury(Hol et al., 2003; Su et al., 2004; Lee et al., 2006; Lee et al., 2008; Fujita et al., 2014). Some researchers explained that overdosed AAV would exert toxic effect on neurons(Khabou et al., 2018; Xiong et al., 2019; Xiang et al., 2021) which may lead to a time-dependent activation of the Gfap promoter. Alternatively, enhanced activity of Gfap promoter may indicate that neuron reverse to an immature transcriptome state upon injury in order to facilitate the neural repair and regeneration process(Poplawski et al., 2020). Moreover, why control virus (AAV-shscramble) seem highly specific to astrocytes but AAV-shPtbp1 that show severe virus leakage to neuron? A recent study explained that the promoter specificity of Gfap is likely cis-regulated by NeuroD1 or some other pro-neural TFs(Wang et al., 2021) Therefore, the control virus may no longer be suitable to serve as a control and thus reliable lineage tracing is indispensable for in vivo cell reprogramming research. 
Secondly, the Cre-activated Loxp-STOP-Loxp (LSL) structure would have leakage expression without Cre recombinase. Even classical LSL structure which has 3-4 repeats of SV40-polyA signal between two Loxp sites can't avoid leakage(Sohal et al., 2009), not to mention the non-classical Loxp-STOP-Loxp (LSL) structure that only has one SV40-polyA signal located in the ORF of Neo/Kana resistance(Qian et al., 2020).

Therefore, without a reliable lineage tracing method, most of the presumed astrocyte-converted neurons were merely endogenous neurons misidentified by unexpected labeling due to virus leakage. The actual AtoN conversion efficiency is probably highly overestimated.

\section{Possible reasons for AtoN conversion failure in vivo}

Compared with the convincing AtoN result in vitro(Qian et al., 2020), why PTBP1 repression fail to convert astrocyte to neuron in vivo? We suppose that AtoN conversion did not even initiate because the no morphologic change of astrocyte could be seen after PTBP1 repression (Fig. 3C \& E) and only subtle changes in gene expression could be seen after astroglial Ptbpl deletion(Blackshaw et al., 2021). The reason why the PTBP1-repressed astrocyte fail to initiate the conversion process may attribute to astrocyte itself and the local microenvironment.

On one thing, the status of astrocyte may greatly influence AtoN conversion outcome since studies found that reactive astrocytes could be converted to neuron more easily than quiescent astrocyte(Guo et al., 2014; Brulet et al., 2017). However, in 6-OHDA lesion model we could not find any sign of AtoN conversion although astrocytes became intensively activated. Reactive astrocytes are highly heterogeneous and can be divided into A1 subtype (pro-inflammatory) and A2 subtype (anti-inflammatory) according to different disease model(Zamanian et al., 2012; Escartin et al., 2021). Whether age-related decrease in neuronal reprogramming efficiency(Qian et al., 2020) is associated with gradual conversion of astrocytes to 
a pro-inflammatory phenotype (A1) with aging(Clarke et al., 2018) and whether anti-inflammatory astrocytes (A2) could be converted to neurons more efficiently need further investigation.

For another, the key difference between in vitro and in vivo is the local microenvironment. Various bioactive molecules either beneficial (neurotrophic factors) or detrimental (inflammatory cytokines) existed in the local microenvironment(Janowska et al., 2019). However, under pathological condition, these secretory factors are usually deleterious (pro-inflammatory cytokines) to the AtoN conversion process and subsequent survival, maturation of the new-born neurons. Unexpectedly, under special pathological condition like ischemia, astrocytes spontaneously initiate a potent neurogenic program after Notch signaling being repressed(Magnusson et al., 2014). Moreover, studies showed that astrocytes from different brain regions have different reprogramming efficiency and different neuron subtype preference (Grande et al., 2013; Liu et al., 2015; Hu et al., 2019; Mattugini et al., 2019). All these findings suggest that the different local environment have different impact on AtoN conversion efficiency and modulating local environment may be critical for enhancing the efficiency of AtoN conversion.

Therefore, only repressing PTBP1 is not enough to initiate cell fate change of astrocyte towards neuron in the mouse brain. The impact of heterogeneity of both astrocyte subtype and local environment on AtoN conversion need further inquiry in the future.

\section{Possibility of neuron replenishment and behavioral recovery after PTBP1 repression}

According Fu's study, using antisense oligonucleotides (ASOs) targeting Ptbpl (Ptbp1-ASO), a fraction of tdTomato-labeled cells in Gfap-creER ${ }^{T M}$ : Rosa-tdTomato mouse were converted to $\mathrm{TH}^{+}$ DAns(Qian et al., 2020). If the astrocyte-to-DAn conversion did not happen, then other cell types rather than astrocyte might contribute to the Ptbpl-ASO-mediated neuron conversion which may explain the DA 
neuron number replenishment, striatal $\mathrm{TH}^{+}$fibers density recovery and behavioral improvement in Fu's study.

The first possible cell source is NSC since PTBP1 is well known to maintain NSC pools(Shibasaki et al., 2013) and is sharply diminished upon neuronal lineage induction(Boutz et al., 2007; Makeyev et al., 2007). Roy Maimon et al reported that using similar Ptbpl-ASO, radial glial-like cells and other GFAP-expressing cells could be converted to neurons(Maimon et al., 2021). Another candidate cell source is oligodendrocyte (OL) which was found to have certain Gfap promoter activity (Behrangi et al., 2020). Weinberg et al. reported that OLs could also be reprogrammed to functional neurons after PTBP1 repression(Weinberg et al., 2017). Therefore, these potent cell types might contribute to the neuron restoration, functional and even behavioral recovery but need further and detailed investigation in the future.

\section{Authors Contribution:}

The manuscript was written with the contributions of all authors. All authors have approved the final version of the manuscript. W. Chen and Q. Zheng conducted most of the experiments. W. Chen and and Q. Huang conducted the literature search and wrote the manuscript. S. Ma and M. Li provided supervision and constructive feedback during manuscript writing. All authors contributed critical revisions to the manuscript.

\section{Funding:}


This study was supported in part by grants from the National Natural Science Foundation of China (U1801681, 81771368, 31871019,81601104), the Key Realm R\&D Program of Guangdong Province (2018B030337001), the Guangdong Provincial Key Laboratory of Brain Function and Disease (2020B1212060024), the National Key R\&D Program of China (2018YFA0108302).

\section{Acknowledgements:}

We thank Qingxing Zhang for his assistance with the animal breeding and genotyping.

\section{Conflict of Interest:}

The authors declare that the research was conducted in the absence of any commercial or financial relationships that could be construed as a potential conflict of interest.

\section{References}

Arenas E (2020) Method to combat Parkinson's disease by astrocyte-to-neuron conversion. Nature 582:489-490.

Behrangi N, Lorenz P, Kipp M (2020) Oligodendrocyte Lineage Marker Expression in eGFP-GFAP Transgenic Mice. J Mol Neurosci.

Blackshaw S, Hoang T, Kim DW, Appel H, Pannullo N, Ozawa M, Zheng S, Yu M, Peachey N, Kim J (2021) Ptbp1 deletion does not induce glia-to-neuron conversion in adult mouse retina and brain. bioRxiv.

Boutz PL, Stoilov P, Li Q, Lin CH, Chawla G, Ostrow K, Shiue L, Ares M, Jr., Black DL (2007) A post-transcriptional regulatory switch in polypyrimidine tract-binding proteins reprograms alternative splicing in developing neurons. Genes \& development 21:1636-1652.

Brulet R, Matsuda T, Zhang L, Miranda C, Giacca M, Kaspar BK, Nakashima K, Hsieh J (2017) NEUROD1 Instructs Neuronal Conversion in Non-Reactive Astrocytes. Stem cell reports 8:1506-1515.

Buffo A, Rite I, Tripathi P, Lepier A, Colak D, Horn AP, Mori T, Gotz M (2008) Origin and progeny of reactive gliosis: A source of multipotent cells in the injured brain. Proceedings of the National Academy of Sciences of 
the United States of America 105:3581-3586.

Clarke LE, Liddelow SA, Chakraborty C, Munch AE, Heiman M, Barres BA (2018) Normal aging induces A1-like astrocyte reactivity. Proceedings of the National Academy of Sciences of the United States of America 115:E1896-E1905.

Escartin C et al. (2021) Reactive astrocyte nomenclature, definitions, and future directions. Nature neuroscience 24:312-325.

Fujita T, Chen MJ, Li B, Smith NA, Peng W, Sun W, Toner MJ, Kress BT, Wang L, Benraiss A, Takano T, Wang S, Nedergaard M (2014) Neuronal transgene expression in dominant-negative SNARE mice. The Journal of neuroscience : the official journal of the Society for Neuroscience 34:16594-16604.

Grande A, Sumiyoshi K, Lopez-Juarez A, Howard J, Sakthivel B, Aronow B, Campbell K, Nakafuku M (2013) Environmental impact on direct neuronal reprogramming in vivo in the adult brain. Nature communications 4:2373.

Guo Z, Zhang L, Wu Z, Chen Y, Wang F, Chen G (2014) In vivo direct reprogramming of reactive glial cells into functional neurons after brain injury and in an Alzheimer's disease model. Cell stem cell 14:188-202.

Hol EM, Roelofs RF, Moraal E, Sonnemans MA, Sluijs JA, Proper EA, de Graan PN, Fischer DF, van Leeuwen FW (2003) Neuronal expression of GFAP in patients with Alzheimer pathology and identification of novel GFAP splice forms. Mol Psychiatry 8:786-796.

Hu X, Qin S, Huang X, Yuan Y, Tan Z, Gu Y, Cheng X, Wang D, Lian XF, He C, Su Z (2019) Region-Restrict Astrocytes Exhibit Heterogeneous Susceptibility to Neuronal Reprogramming. Stem cell reports 12:290-304.

Janowska J, Gargas J, Ziemka-Nalecz M, Zalewska T, Buzanska L, Sypecka J (2019) Directed glial differentiation and transdifferentiation for neural tissue regeneration. Experimental neurology 319:112813.

Jiang Y, Wang Y, Huang Z (2021) Targeting PTB as a One-Step Procedure for In Situ Astrocyte-to-Dopamine Neuron Reprogramming in Parkinson's Disease. Neurosci Bull 37:430-432.

Khabou H, Cordeau C, Pacot L, Fisson S, Dalkara D (2018) Dosage Thresholds and Influence of Transgene Cassette in Adeno-Associated Virus-Related Toxicity. Hum Gene Ther 29:1235-1241.

Kretzschmar K, Watt FM (2012) Lineage tracing. Cell 148:33-45.

Lee Y, Su M, Messing A, Brenner M (2006) Astrocyte heterogeneity revealed by expression of a GFAP-LacZ transgene. Glia 53:677-687.

Lee $Y$, Messing A, Su M, Brenner M (2008) GFAP promoter elements required for region-specific and astrocyte-specific expression. Glia 56:481-493.

Lentini C, d'Orange M, Marichal N, Trottmann MM, Vignoles R, Foucault L, Verrier C, Massera C, Raineteau O, Conzelmann KK, Rival-Gervier S, Depaulis A, Berninger B, Heinrich C (2021) Reprogramming reactive glia into interneurons reduces chronic seizure activity in a mouse model of mesial temporal lobe epilepsy. Cell stem cell.

Liu Y, Miao Q, Yuan J, Han S, Zhang P, Li S, Rao Z, Zhao W, Ye Q, Geng J, Zhang X, Cheng L (2015) Ascl1 Converts Dorsal Midbrain Astrocytes into Functional Neurons In Vivo. The Journal of neuroscience : the official journal of the Society for Neuroscience 35:9336-9355.

Magnusson JP, Goritz C, Tatarishvili J, Dias DO, Smith EM, Lindvall O, Kokaia Z, Frisen J (2014) A latent neurogenic program in astrocytes regulated by Notch signaling in the mouse. Science 346:237-241.

Maimon R, Chillon-Marinas C, Snethlage CE, Singhal SM, McAlonis-Downes M, Ling K, Rigo F, Bennett CF, Da Cruz S, Hnasko TS, Muotri AR, Cleveland DW (2021) Therapeutically viable generation of neurons with antisense oligonucleotide suppression of PTB. Nature neuroscience 24:1089-1099.

Makeyev EV, Zhang J, Carrasco MA, Maniatis T (2007) The MicroRNA miR-124 promotes neuronal differentiation by triggering brain-specific alternative pre-mRNA splicing. Mol Cell 27:435-448. 
Mattugini N, Bocchi R, Scheuss V, Russo GL, Torper O, Lao CL, Gotz M (2019) Inducing Different Neuronal Subtypes from Astrocytes in the Injured Mouse Cerebral Cortex. Neuron 103:1086-1095 e1085.

Niu W, Zang T, Zou Y, Fang S, Smith DK, Bachoo R, Zhang CL (2013) In vivo reprogramming of astrocytes to neuroblasts in the adult brain. Nat Cell Biol 15:1164-1175.

Poplawski GHD, Kawaguchi R, Van Niekerk E, Lu P, Mehta N, Canete P, Lie R, Dragatsis I, Meves JM, Zheng B, Coppola G, Tuszynski MH (2020) Injured adult neurons regress to an embryonic transcriptional growth state. Nature 581:77-82.

Qian C, Dong B, Wang XY, Zhou FQ (2021) In vivo glial trans-differentiation for neuronal replacement and functional recovery in central nervous system. FEBS J 288:4773-4785.

Qian H, Kang X, Hu J, Zhang D, Liang Z, Meng F, Zhang X, Xue Y, Maimon R, Dowdy SF, Devaraj NK, Zhou Z, Mobley WC, Cleveland DW, Fu XD (2020) Reversing a model of Parkinson's disease with in situ converted nigral neurons. Nature 582:550-556.

Rivetti di Val Cervo P, Romanov RA, Spigolon G, Masini D, Martin-Montanez E, Toledo EM, La Manno G, Feyder M, Pifl C, Ng YH, Sanchez SP, Linnarsson S, Wernig M, Harkany T, Fisone G, Arenas E (2017) Induction of functional dopamine neurons from human astrocytes in vitro and mouse astrocytes in a Parkinson's disease model. Nature biotechnology 35:444-452.

Robel S, Berninger B, Gotz M (2011) The stem cell potential of glia: lessons from reactive gliosis. Nature reviews Neuroscience 12:88-104.

Sanz E, Yang L, Su T, Morris DR, McKnight GS, Amieux PS (2009) Cell-type-specific isolation of ribosome-associated mRNA from complex tissues. Proceedings of the National Academy of Sciences of the United States of America 106:13939-13944.

Shibasaki T, Tokunaga A, Sakamoto R, Sagara H, Noguchi S, Sasaoka T, Yoshida N (2013) PTB deficiency causes the loss of adherens junctions in the dorsal telencephalon and leads to lethal hydrocephalus. Cereb Cortex 23:1824-1835.

Shimada IS, LeComte MD, Granger JC, Quinlan NJ, Spees JL (2012) Self-renewal and differentiation of reactive astrocyte-derived neural stem/progenitor cells isolated from the cortical peri-infarct area after stroke. The Journal of neuroscience : the official journal of the Society for Neuroscience 32:7926-7940.

Sirko S et al. (2013) Reactive glia in the injured brain acquire stem cell properties in response to sonic hedgehog. [corrected]. Cell stem cell 12:426-439.

Sohal VS, Zhang F, Yizhar O, Deisseroth K (2009) Parvalbumin neurons and gamma rhythms enhance cortical circuit performance. Nature 459:698-702.

Srinivasan R, Lu TY, Chai H, Xu J, Huang BS, Golshani P, Coppola G, Khakh BS (2016) New Transgenic Mouse Lines for Selectively Targeting Astrocytes and Studying Calcium Signals in Astrocyte Processes In Situ and In Vivo. Neuron 92:1181-1195.

Su M, Hu H, Lee Y, D'Azzo A, Messing A, Brenner M (2004) Expression specificity of GFAP transgenes. Neurochem Res 29:2075-2093.

Torper O, Gotz M (2017) Brain repair from intrinsic cell sources: Turning reactive glia into neurons. Progress in brain research 230:69-97.

Wan J, Zhao XF, Vojtek A, Goldman D (2014) Retinal injury, growth factors, and cytokines converge on beta-catenin and pStat3 signaling to stimulate retina regeneration. Cell Rep 9:285-297.

Wang LL, Serrano C, Zhong X, Ma S, Zou Y, Zhang CL (2021) Revisiting astrocyte to neuron conversion with lineage tracing in vivo. Cell.

Weinberg MS, Criswell HE, Powell SK, Bhatt AP, McCown TJ (2017) Viral Vector Reprogramming of Adult Resident Striatal Oligodendrocytes into Functional Neurons. Molecular therapy : the journal of the 
American Society of Gene Therapy 25:928-934.

Wu Z, Parry M, Hou XY, Liu MH, Wang H, Cain R, Pei ZF, Chen YC, Guo ZY, Abhijeet S, Chen G (2020) Gene therapy conversion of striatal astrocytes into GABAergic neurons in mouse models of Huntington's disease. Nature communications 11:1105.

Xiang Z, Xu L, Liu M, Wang Q, Li W, Lei W, Chen G (2021) Lineage tracing of direct astrocyte-to-neuron conversion in the mouse cortex. Neural Regen Res 16:750-756.

Xiong W, Wu DM, Xue Y, Wang SK, Chung MJ, Ji X, Rana P, Zhao SR, Mai S, Cepko CL (2019) AAV cis-regulatory sequences are correlated with ocular toxicity. Proceedings of the National Academy of Sciences of the United States of America 116:5785-5794.

Zamanian JL, Xu L, Foo LC, Nouri N, Zhou L, Giffard RG, Barres BA (2012) Genomic analysis of reactive astrogliosis. The Journal of neuroscience : the official journal of the Society for Neuroscience 32:6391-6410.

Zhou H et al. (2020) Glia-to-Neuron Conversion by CRISPR-CasRx Alleviates Symptoms of Neurological Disease in Mice. Cell 181:590-603.e516. 


\section{Graphic abstract}

AAV-shPtbp1-induced GFP+ dopaminergic neuron were not orginated from astrocyte

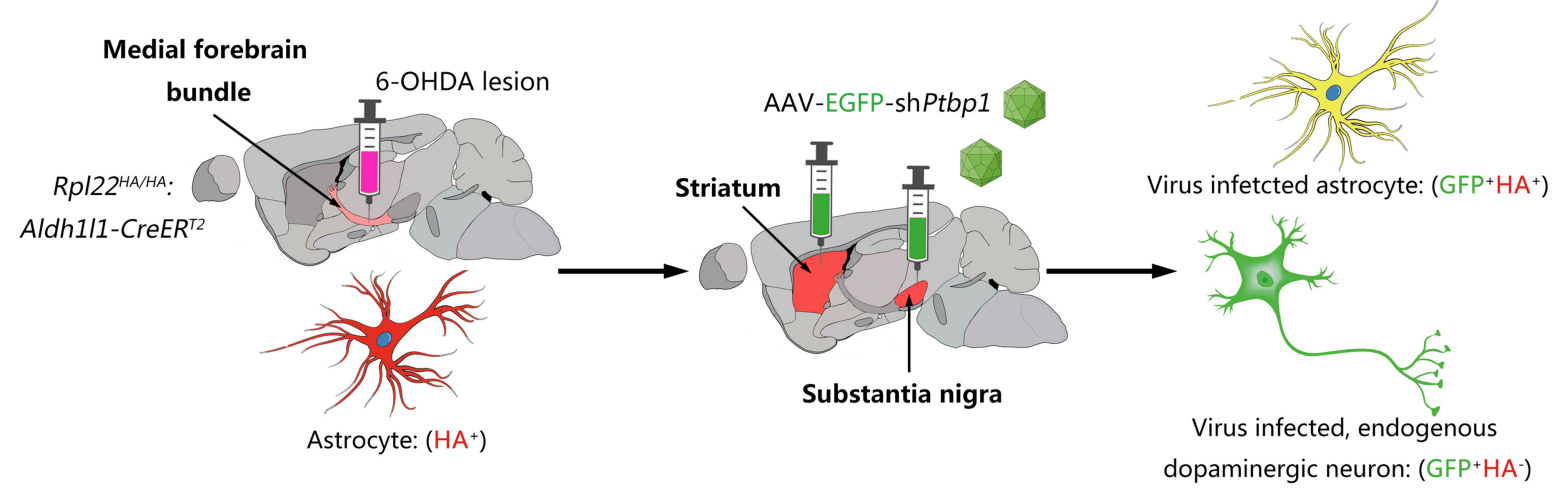

\section{Highlights}

-AAV-shPtbp1 rapidly and efficiently induces viral-reporter-labeled DAns in mouse brain under physiological condition

-Viral-reporter-positive DAns are not originated from PTBP1 repressed and lineage traced reactive astrocytes in a mouse PD model 

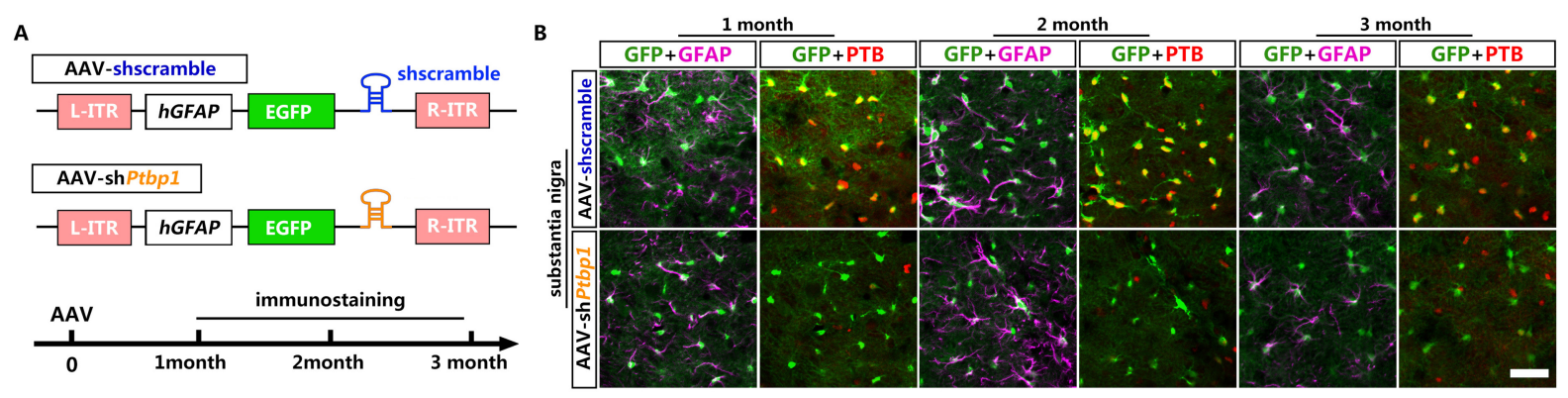

C
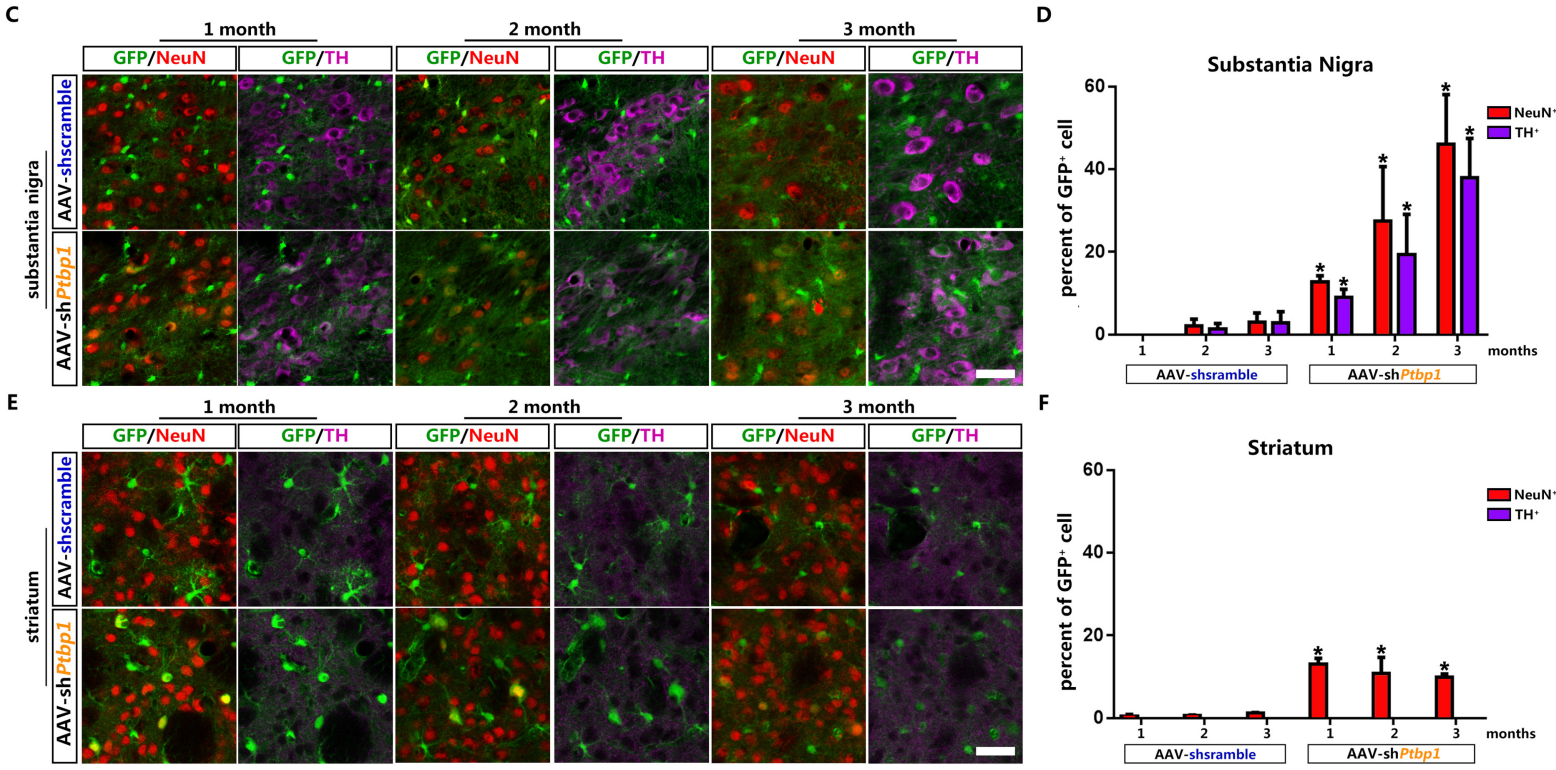

Fig. 1 Reporter ${ }^{+}$neurons were gradually induced in the substantia nigra and the striatum after

\section{astroglial PTBP1 repression by AAV-shPtbp1.}

(A) Schematic of AAV-shPtbp1 and AAV-shscramble vector design and the experimental design. (B,C,E)

Representative images of brain slices co-stained GFP (green) with GFAP (purple) or PTBP1 (red) in (B),

with NeuN (red) or TH (purple) in (C \& E) at indicated time points after AAV-shPtbpl or AAV-shscramble delivery. Scale bar, $50 \mu \mathrm{m}$. (D \& F) Number of $\mathrm{GFP}^{+}$cells in the substantia nigra (D) and in the striatum (F) that show positive staining for NeuN (red) and TH (purple). $\mathrm{n}=3$ biological repeats. Data are presented as mean \pm SEM. *Indicates significant difference between AAV-shPtbp1 and AAV-scramble. 
A

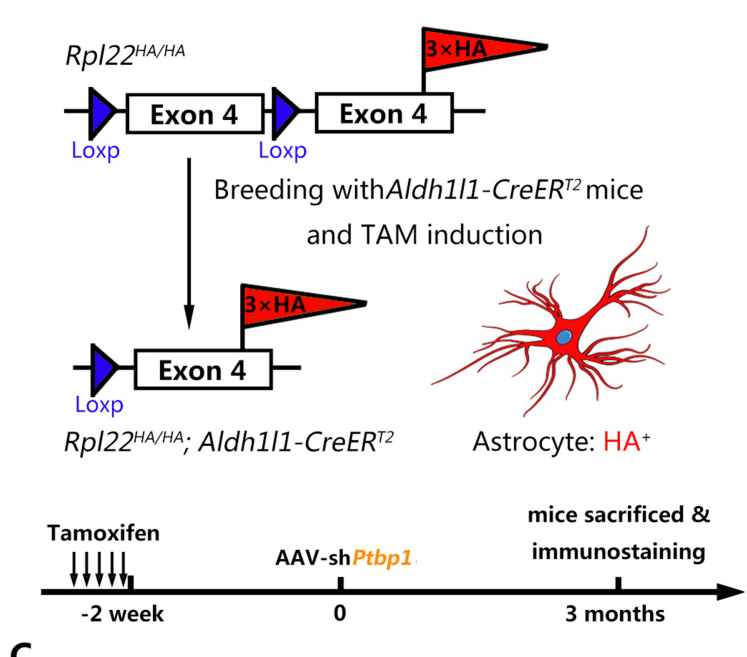

B

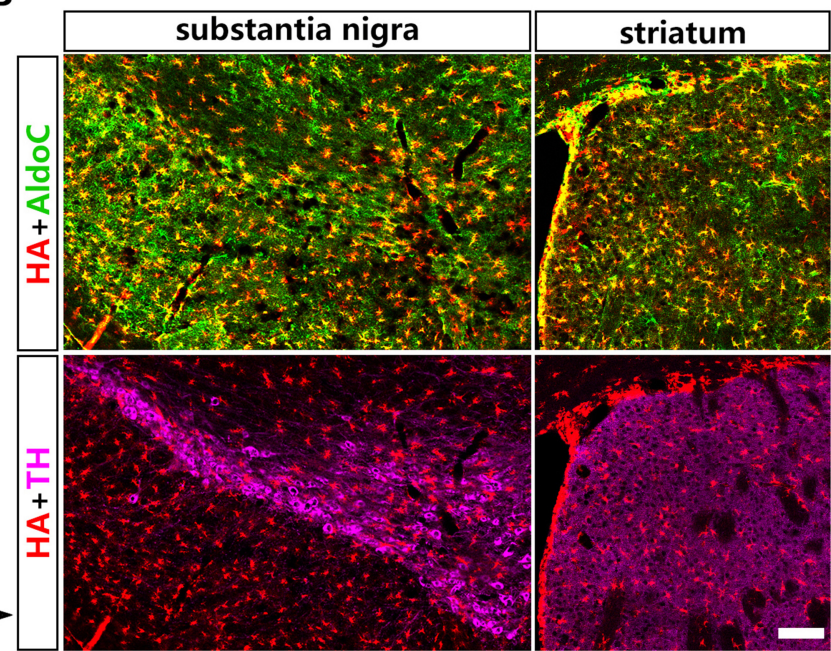

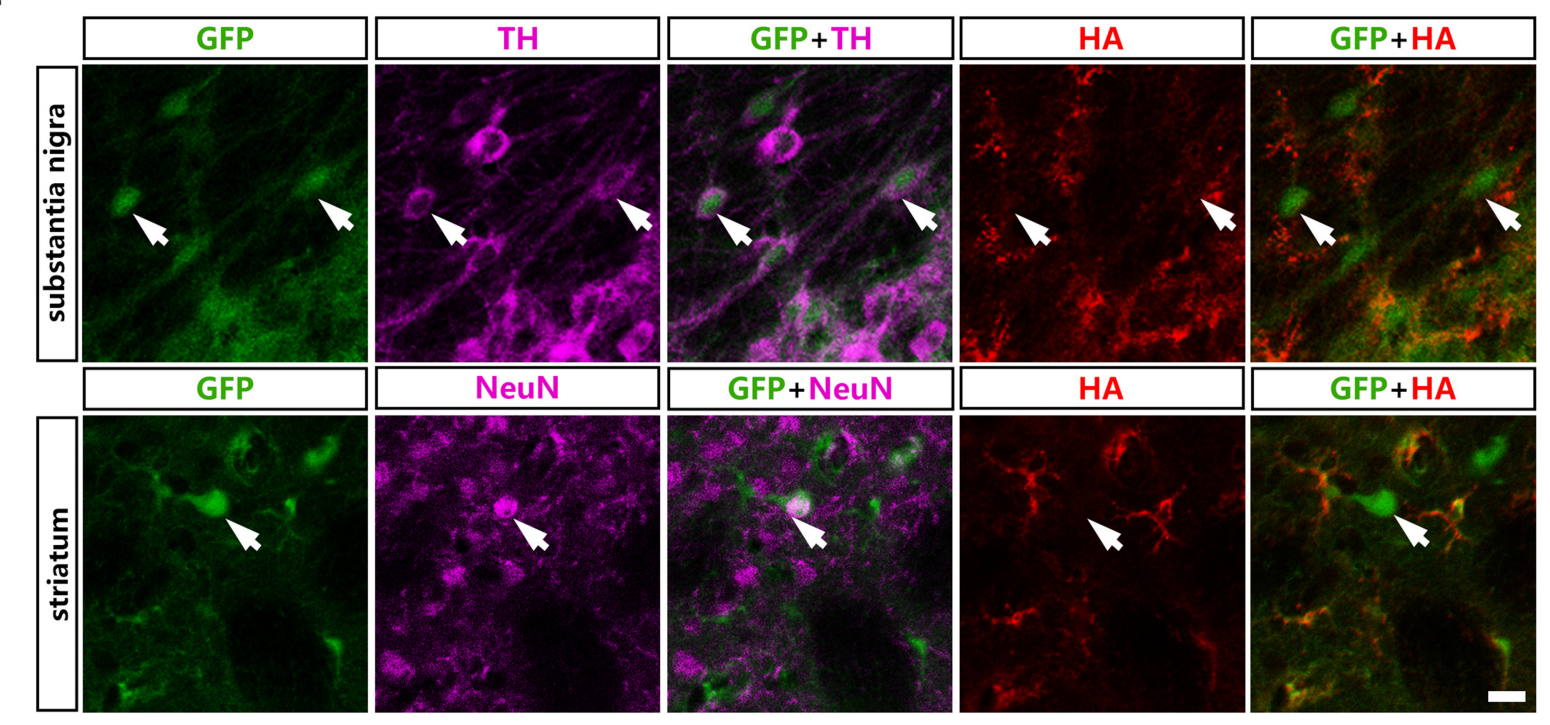

Fig. 2 AAV-shPtbp1-induced reporter ${ }^{+}$neurons including DAns were not derived from $\mathrm{HA}^{+}$quiescent astrocytes.

(A) Schematic of breeding strategy of Aldh1l1-CreER ${ }^{T 2}$ :Ribotag mice and experimental design. (B) Representative images of substantia nigra or striatum of $A l d h 1 l 1-C r e E R^{T 2}$ :Ribotag mice co-stained HA (red) with pan-astrocyte marker AldoC (green) and TH (purple) 2 weeks after TAM administration. Scale bar, $100 \mu \mathrm{m}$. (C) Representative images of brain slices co-stained GFP (green), HA (red) with TH (purple) in the substantia nigra and with NeuN (purple) in the striatum 3 months after AAV-shPtbpl delivery. Scale bar, $75 \mu \mathrm{m}$. 
A

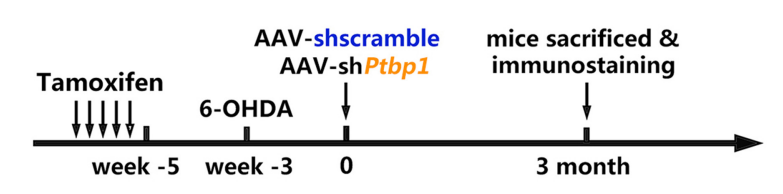

B

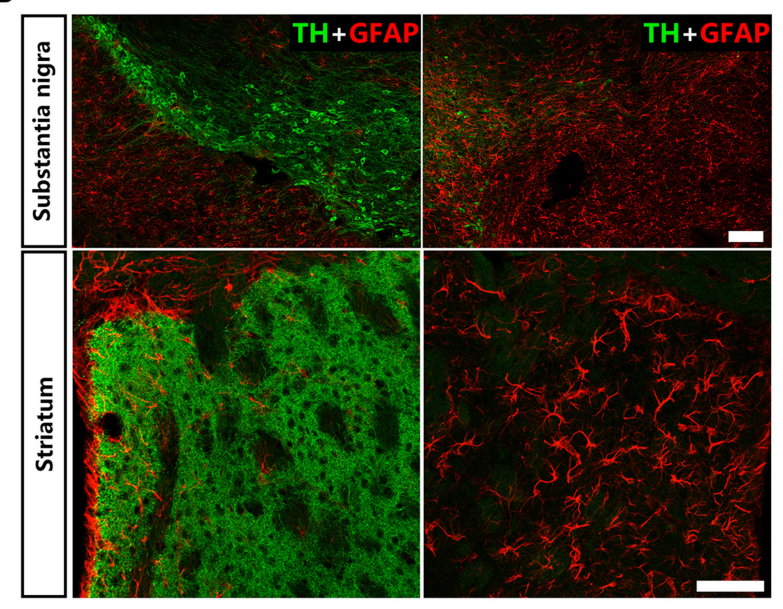

C

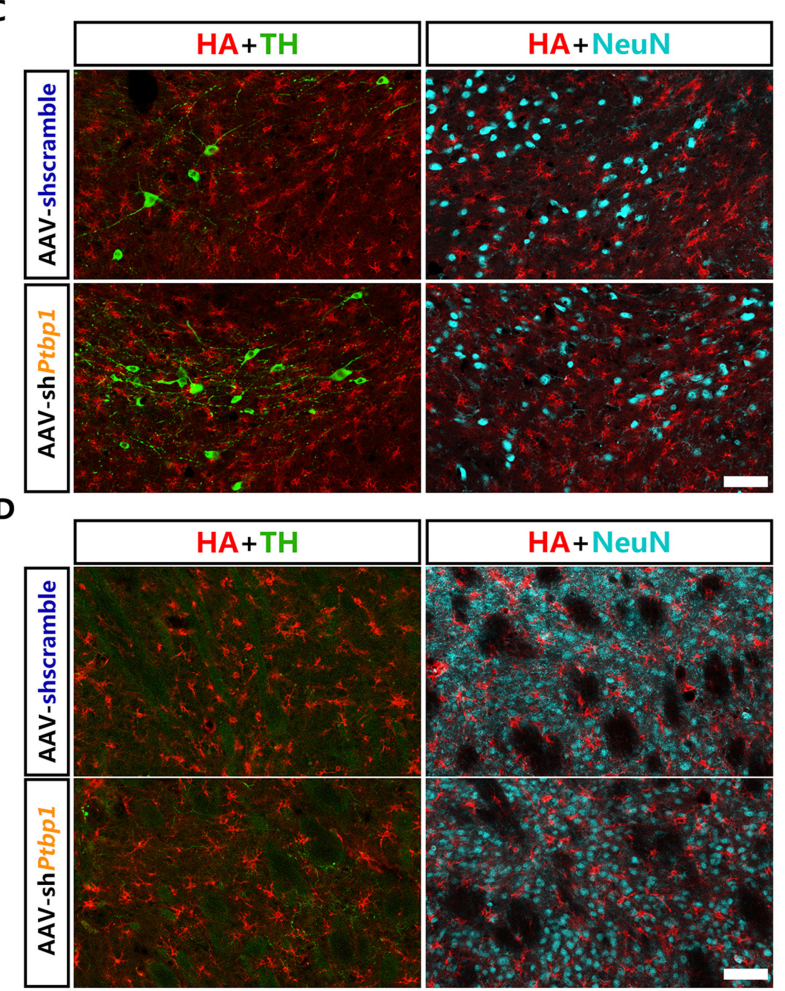

Fig. 3 No neuron including DAn co-staining HA can be detected after astroglial PTBP1 repression in

\section{6-OHDA model}

(A) Schematic of experimental design. (B) Representative images of substantia nigra or striatum after 6-OHDA lesion co-stained with TH (green) and GFAP (red). Scale bar, $100 \mu \mathrm{m}$ for the substantia nigra and $50 \mu \mathrm{m}$ for the striatum. (C,D) Representative images of HA (red), TH (green) and NeuN (cyan) co-stained substantia nigra (C) and striatum (D) 3 months after AAV-shPtbpl or AAV-shscramble delivery in 6-OHDA model. Scale bar, $25 \mu \mathrm{m}$. 